\section{Pilotstudie: Alphablocker hilft auch bei vorzeitiger Ejakulation}

\begin{abstract}
Alphablocker werden bei Symptomen der unteren Harnwege (LUTS) erfolgreich eingesetzt, allerdings wirken die mit der Einnahme verbundenen Ejakulationsprobleme in diesem Zusammenhang störend. Anders bei Patienten mit Ejaculatio praecox: Hier, so spekulierten japanische Forscher, könnte man sich die ejakulationshemmende Wirkung zunutze machen.
\end{abstract}

apanische Urologen haben erstmals antagonisten Silodosin, ursprünglich ein Medikament zur Behandlung von Symptomen der unteren Harnwege (LUTS), auch bei Ejaculatio praecox wirkt. Für die Pilotstudie wurden acht Männer ausgewählt, die nach DSM-IV-Kriterien unter vorzeitiger Ejakulation litten und bei denen die Lebensqualität deutlich beeinträchtigt war. Bei sechs Patienten bestand zusätzlich eine erektile Dysfunktion, die mit Phosphodiesterasehemmern behandelt wurde. Das durchschnittliche Alter lag bei etwas über 54 Jahren.

Die Männer nahmen über zwei Monate jeweils zwei Stunden vor dem (geplanten) Geschlechtsverkehr 4 mg Silodosin ein. Als Bewertungsmaßstab für den Therapieerfolg wurden die intravaginale Ejaku- lationsverzögerungszeit (IELT) sowie die Clinical Global Impression Scale (CGIC) herangezogen.

Im Durchschnitt verlängerte sich die IELT von 3,4 auf 10,1 Minuten ( $p=0,003)$. Sechs Patienten (75\%) gaben an, die Symptome seien „besser“ oder „viel besser“ geworden, zwei schilderten nur eine "leichte“ Verbesserung. Die Teilnehmer konnten nach der Behandlung ihre Ejakulation signifikant besser kontrollieren $(p=0,003)$, sechs Männer empfanden den Geschlechtsverkehr als deutlich befriedigender als zuvor, und bei ebenfalls sechs Männern besserte sich auch der Leidensdruck.

Wie erwartet zeigte sich unter Behandlung bei der Mehrzahl der Patienten (87,5\%) ein leichtes Missempfinden bei der Ejakulation, was die Männer aber of- fenbar nicht sehr beeinträchtigte. Zwei Patienten waren nicht imstande zu ejakulieren, drei $(37,5 \%)$ berichteten über ein vermindertes Samenvolumen. Die Autoren hatten die Patienten im Vorfeld über diese möglichen Effekte aufgeklärt. Substanzklassenspezifische Nebenwirkungen wie orthostatische Hypotonie traten in der kleinen Teilnehmergruppe nicht auf. Die Autoren führen dies auf die Selektivität von Silodosin zurück, das speziell am a1A-Adrenozeptor angreift.

Fazit: Die Daten unterstützen die Anwendung von Silodosin bei Patienten mit Ejaculatio praecox. Allerdings sollten die Patienten vor Anwendung auf mögliche Nebeneffekte wie Anejakulation, Missempfindungen beim Orgasmus und vermindertes Samenvolumen aufmerksam gemacht werden. Es sei nicht auszuschließen, dass diese bei breitem Einsatz die Lebensqualität beeinträchtigen könnten. Weitere placebokontrollierte Studien sollen folgen.

Dr. Elke Oberhofer

Sato $Y$ et al. Silodosin and its potential for treating premature ejaculation: A preliminary report. Int J Urol 2012; 19: 268-72

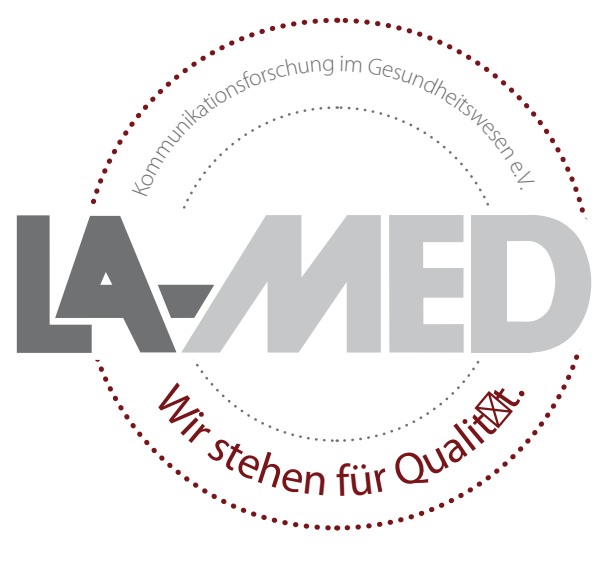
medizinischen Fachpresse in den n囚chsten Wochen zusammen mit tnsemnid 